'Departamento de Nutrición, Diabetes y Metabolismo, Pontificia Universidad Católica de Chile. Santiago, Chile. ${ }^{2}$ Departamento de Gastroenterología, Pontificia Universidad Católica de Chile. Santiago, Chile.

Trabajo no recibió financiamiento. Los autores declaran no tener conflictos de interés.

Recibido el 29 de agosto de 2019, aceptado el 10 de agosto de 2020.

Correspondencia a: Dra. Paulina Jofré Departamento de Nutrición, Diabetes y Metabolismo, Pontificia Universidad Católica de Chile. Santiago, Chile. pejofre@uc.cl

\section{Tratamiento de quilotórax y quiloascitis refractaria en paciente cirrótico. Caso clínico}

\author{
PAULINA JOFRÉ ${ }^{1}$, BRUNO GRASSI $^{1}$, CARLOS BENÍTEZ $^{2}$
}

\section{Management of chylous ascites and chylothorax. Report of one case}

Chylous Ascites (CA) and chylothorax (CTx) are associated with obstruction, disruption or insufficiency of the lymphatic system. We report a 68-year-old male, with a history of alcoholic cirrhosis, who had recurrent events of CTx and $C A$. After a complete study, no other etiologies other than portal hypertension were found. Therapy with diuretics, nothing per mouth, parenteral feeding plus octreotide did not relieve symptoms. A transjugular intrahepatic portosystemic shunt (TIPS) was successfully placed and pleural effusion subsided. This case shows that CA and CTx can be caused by portal hypertension and they may subside employing a multimodal management strategy.

(Rev Med Chile 2020; 148: 1202-1206)

Key words: Chylous Ascites; Hypertension, Portal; Liver Cirrhosis; Nutritional Support; Portasystemic Shunt, Transjugular Intrahepatic.
L a ascitis quilosa (AQ) y el quilotórax (QT) corresponden a la acumulación de quilo en la cavidad peritoneal y el espacio pleural, respectivamente. Se diagnostican por una concentración de triglicéridos $>200 \mathrm{mg} / \mathrm{dL}$ para el caso del líquido ascítico ${ }^{2,3} \mathrm{y} \geq 110 \mathrm{mg} / \mathrm{dL}$ para el fluido pleural ${ }^{2}$.

Ambas entidades se asocian a obstrucción o disrupción del sistema linfático (cisterna de quilo o ducto torácico). Sus causas son secundarias a trauma, cirugía o condiciones médicas tales como neoplasias, infecciones (tuberculosis), insuficiencia cardiaca, anomalías congénitas, cirrosis con hipertensión portal e idiopáticas ${ }^{1,2}$. La cirrosis hepática es una causa frecuente de AQ en occidente, estando presente en $0,5 \%-1 \%$ de los casos ${ }^{3}$. El mecanismo fisiopatológico estaría asociado a un aumento masivo del flujo linfático y la incapacidad del sistema linfático de conducirlo en el contexto de hipertensión portal ${ }^{3}$.

El quilo está compuesto por triglicéridos, albúmina, electrolitos y linfocitos. Por ello, la pérdida continua de quilo conlleva un deterioro del estado nutricional e inmunológico ${ }^{4}$.

Existen distintos enfoques de tratamiento, sin un claro consenso en su manejo, el cual incluye estrategias nutricionales, reducción del flujo linfático, cirugías, terapias endolinfáticas, entre otras, dependiendo de la causa subyacente ${ }^{5}$.

En el siguiente caso se expone una estrategia de tratamiento multimodal de quiloascitis y quilotórax en un paciente con cirrosis.

\section{Caso clínico}

Presentamos el caso de un paciente de sexo masculino de 68 años, con antecedentes de cirrosis secundaria al consumo de alcohol, Child Pugh B, MELD-Na 15 puntos, várices esofágicas en profilaxis primaria con ligadura endoscópica, hipertensión arterial y diabetes mellitus tipo 2, con historia de disnea de un mes de evolución $y$ ascitis refractaria al uso de diuréticos a dosis 


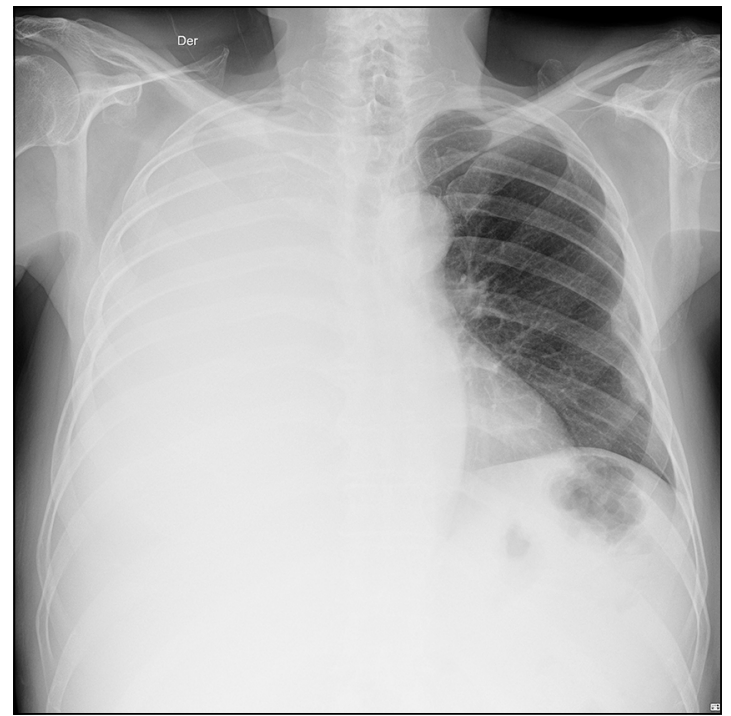

Figura 1a. Radiografía de tórax en primera hospitalización.

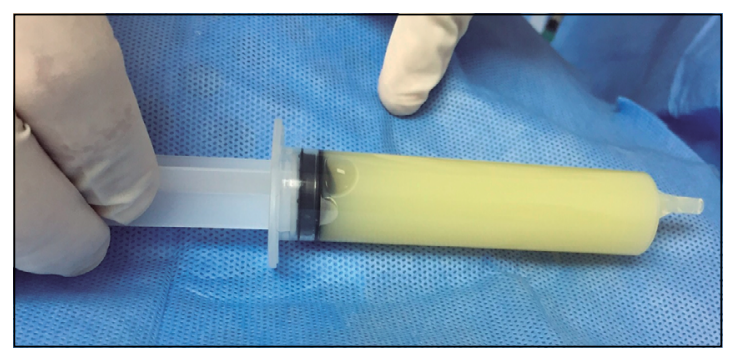

Figura 1b. Líquido pleural de aspecto quiloso.

máxima (furosemida $160 \mathrm{mg} /$ día y espironolactona $400 \mathrm{mg} /$ día). Se solicitó radiografía de tórax que mostró velamiento completo del hemitórax derecho (Figura 1a).

$\mathrm{Al}$ momento del ingreso hospitalario presentaba los siguientes exámenes en sangre: creatinina 4,2 $\mathrm{mg} / \mathrm{dl}$, sodio: $132 \mathrm{mEq} / \mathrm{L}$; potasio $5,9 \mathrm{mEq} / \mathrm{l}$; cloro $92 \mathrm{mE} / \mathrm{L}, \mathrm{GPT} 17 \mathrm{U} / \mathrm{L}, \mathrm{GOT} 33 \mathrm{U} / \mathrm{L}, \mathrm{GGT}$ $127 \mathrm{U} / \mathrm{L}, \mathrm{FA} 119 \mathrm{U} / \mathrm{L}$, bilirrubina total $1,36 \mathrm{mg} / \mathrm{dl}$, bilirrubina directa $0,79 \mathrm{mg} / \mathrm{dl}$, INR 1,2 , albúmina 3,3 g/dl, triglicéridos $110 \mathrm{mg} / \mathrm{dL}, \mathrm{LDH} 179 \mathrm{U} / \mathrm{L}$ glucosa $239 \mathrm{mg} / \mathrm{dL}$. Se realizó estudio de líquidos ascítico y pleural, con triglicéridos elevados hasta $439 \mathrm{mg} / \mathrm{dL}$ y $592 \mathrm{mg} / \mathrm{dL}$, respectivamente (Tabla 1 y Figura 1b).

En el contexto de un líquido ascítico dependiente de hipertensión portal se diagnosticó una
AQ y un QT secundario a cirrosis. Se trató con toracocentesis y paracentesis evacuadoras asociado a reposición de albúmina, presentando mejoría de la sintomatología respiratoria, con desaparición del velamiento de hemitórax derecho en la radiografía de control. La función renal se recuperó tras la suspensión de los diuréticos durante la hospitalización. La tomografía computada (TAC) de tórax, abdomen y pelvis no mostró evidencias de neoplasia ni lesiones evidentes del ducto torácico o de la cisterna de quilo. La endoscopia digestiva alta y la colonoscopia fueron normales. Al alta se indicó régimen hipograso e hiposódico asociado a administración de triglicéridos de cadena media (MCT) vía oral y se reiniciaron los diuréticos en dosis bajas.

Posteriormente, el paciente reingresó en tres oportunidades por disnea secundaria a QT en un período de tres meses, a pesar de estar con el tratamiento descrito. En el último ingreso presentó falla respiratoria secundaria a QT masivo, requiriendo la instalación de emergencia de un tubo de drenaje pleural. Dado cuadro de difícil manejo, se decidió suspender alimentación por vía oral, apoyándolo con nutrición parenteral (NPT) $(1,5$ g proteínas $/ \mathrm{kg}$ y $26 \mathrm{kcal} / \mathrm{kg}$ ), asociado a octreótide (100 mcg cada $8 \mathrm{~h} \mathrm{sc}$ ) progresando paulatinamente a nutrición enteral con fórmula rica en MCT, con buena respuesta, evolucionando con disminución del débito del drenaje pleural (Figura 1c) y la

Tabla 1. Características del líquido ascítico y pleural al diagnóstico

\begin{tabular}{|lcc|}
\hline & $\begin{array}{c}\text { Líquido } \\
\text { ascítico }\end{array}$ & $\begin{array}{c}\text { Líquido } \\
\text { pleural }\end{array}$ \\
\hline Proteínas & $3,4 \mathrm{~g} / \mathrm{dL}$ & $4,5 \mathrm{~g} / \mathrm{dL}$ \\
\hline Albúmina & $1,6 \mathrm{~g} / \mathrm{dL}$ & $2,5 \mathrm{~g} / \mathrm{dL}$ \\
\hline LDH & $70 \mathrm{U} / \mathrm{L}$ & $101 \mathrm{U} / \mathrm{L}$ \\
\hline Glucosa & $131 \mathrm{mg} / \mathrm{dL}$ & $143 \mathrm{mg} / \mathrm{dL}$ \\
\hline Gradiente albúmina & $2,6 \mathrm{~g} / \mathrm{dL}$ & $1,6 \mathrm{~g} / \mathrm{dL}$ \\
\hline Triglicéridos & $439 \mathrm{mg} / \mathrm{dL}$ & $592 \mathrm{mg} / \mathrm{dL}$ \\
\hline Recuento celular & $700 \times \mathrm{mm} m^{3}$ & $78 \times \mathrm{mm}{ }^{3}$ \\
\hline Glóbulos blancos & $636 \times \mathrm{mm} m^{3}$ & $71 \times \mathrm{mm}$ \\
\hline PMN & $0 \%$ & $1 \%$ \\
\hline Cultivos & Negativo & Negativo \\
\hline ADA & $6 \mathrm{U} / \mathrm{L}$ & - \\
\hline
\end{tabular}




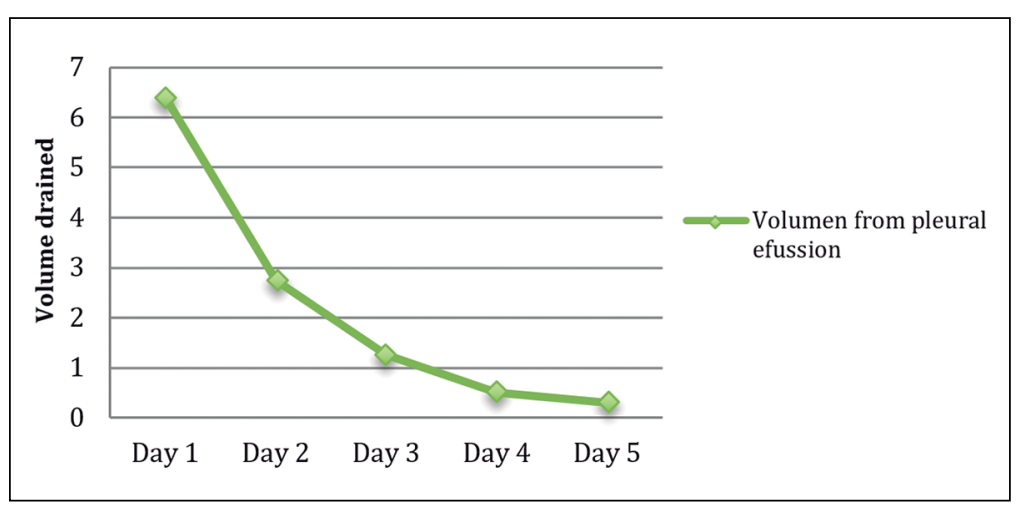

Figura 1c. Volumen drenado de líquido pleural (L) durante la terapia con nutrición parenteral total y ocreótide.
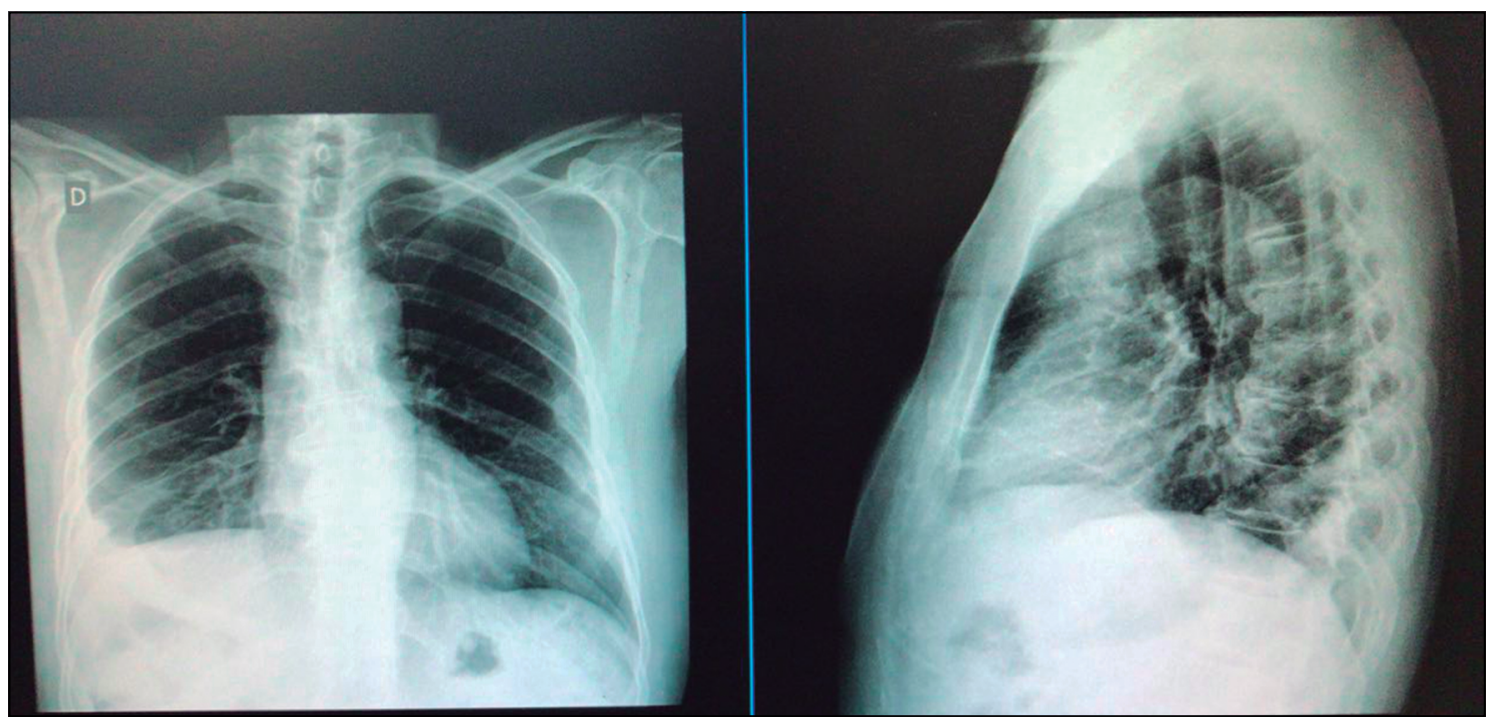

Figura 1d. Radiografía de tórax 1 año posterior a la terapia.

concentración de triglicéridos en el líquido pleural hasta valores normales $(110 \mathrm{mg} / \mathrm{dL})$.

A pesar de la buena respuesta, se objetivó la reproducción de ascitis e hidrotórax tras el retiro del drenaje pleural ( $\sin$ componente quiloso) requiriendo evacuaciones sucesivas. Por lo anterior, se decidió la instalación de derivación portosistémica intrahepática transyugular (TIPS, por su nombre en inglés: transyugular intrahepatic portosystemic shunt), logrando una disminución de la gradiente de presión portal de $13 \mathrm{mmHg}$ (pre TIPS) a $3 \mathrm{mmHg}$ (post TIPS), sin desarrollo de encefalopatía hepática, ni reproducción de ascitis ni del hidrotórax, asociado a el uso de diuréticos a dosis bajas (espironolactona $50 \mathrm{mg} /$ día), restric- ción de sal y administración de MCT tras 1 año de seguimiento (Figura 1d).

\section{Discusión}

La QA puede observarse en cirrosis con una frecuencia baja. El QT es menos frecuente, tiene características de transudado y cuando está presente se asocia a QA. Se postula que el mecanismo de formación del QT en estos pacientes sería por una translocación de fluido a través de defectos del diafragma ${ }^{6}$.

Ante la presencia de fluidos quilosos, el estudio debe dirigirse a detectar la etiología. Así, se requie- 
re descartar neoplasias, trauma, investigar cirugías previas y patologías infecciosas ${ }^{7}$, permitiendo instaurar un tratamiento específico y estimar el pronóstico de cada caso. Una técnica descrita para el estudio del sistema linfático es la linfografía con resonancia magnética (linfoRNM). Esta proporciona información sobre los vasos linfáticos y linfonodos mediante la visualización en tiempo real del flujo linfático contrastado. Esta herramienta visualiza el sistema linfático desde extremidades inferiores al ducto torácico. Sin embargo, el hígado y los sistemas linfáticos intestinales están fuera de las vías de contraste y no puede ser visualizado ${ }^{8}$, por lo que en este caso no fue utilizado.

El principal impacto de estas entidades clínicas en las personas con cirrosis está asociado a las consecuencias originadas por la pérdida de quilo (desnutrición, infecciones, sepsis). Es por ello que se requiere de un diagnóstico y manejo oportuno. El tratamiento debe ser individualizado, ajustado al grado de gravedad, carencias específicas y respuesta al manejo iniciado.

En el caso expuesto, se detecta como etiología gatillante la cirrosis y la presencia de hipertensión portal. Inicialmente se realizó un tratamiento conservador con diuréticos y dieta hipograsa, suplementado con MCT. El soporte nutricional permite reponer la pérdida de fluidos, electrolitos y nutrientes, evitando la desnutrición y disminuyendo la producción de quilo. El flujo de quilo en el conducto torácico varía, dependiendo, entre otros factores, del contenido lipídico de la dieta. Dado que $95 \%$ de la grasa dietética está compuesta por triglicéridos de cadena larga (LCT), el tratamiento nutricional está basado en la limitación de su ingesta, ya que estos forman parte de los quilomicrones que son transportados a través de la linfa. Para poder suministrar los requerimientos calóricos con este tipo de dietas restrictivas, se emplean suplementos de MCT. Estos se absorben en forma rápida y eficiente, a nivel del intestino delgado. Son transportados hacia el hígado por vía portal, sin ser incorporados a quilomicrones y son un substrato rápido de $\beta$ oxidación a nivel mitocondrial. Su rápida absorción en la circulación portal reduce la producción y el flujo de quilo9. Con el empleo de estas medidas se ha reportado el cierre espontáneo de fístulas o defectos de vasos linfáticos en $75 \%-80 \%{ }^{10-12}$. En relación al uso de NPT, se describe cuando las medidas previas han fracasado. Con esta se logra el reposo digestivo absoluto, disminuyendo la producción de linfa desde $220 \mathrm{~mL} / \mathrm{kg} / \mathrm{h}$ a $1 \mathrm{~mL} / \mathrm{kg} / \mathrm{h}^{13}$. Las tasas de éxito reportadas son $54 \%-81 \%$, asociándose a un mayor porcentaje de cierre espontáneo de la fístula y menor duración del tratamiento médico ${ }^{14}$. En cuanto al uso de análogos de somatostatina (octreótide) en el tratamiento de QT y QA, toda la evidencia disponible se limita a casos aislados o series de casos, no existiendo ensayos clínicos que contrasten su eficacia y seguridad ${ }^{9}$. El mecanismo por el cual disminuye la producción de quilo se explica por inhibición en la absorción de las grasas, aumentando su excreción por las heces, disminuyendo la producción de ácido gástrico, pepsina y factores intrínsecos que degradan las grasas en formas más absorbibles, disminución de la presión portal y el flujo sanguíneo esplácnico ${ }^{15}$.

A pesar de la disminución de QT, el paciente evolucionó con recurrencia de derrame pleural y falla respiratoria, requiriendo la instalación de un drenaje pleural. Si bien el uso de esta medida está teóricamente contraindicado por el riesgo aumentado de pérdidas de linfocitos, proteínas e inmunoglobulinas presentes en el quilo, se ha reportado su utilidad en casos de QT de origen maligno y benigno, sin desarrollar complicaciones o efectos adversos ${ }^{16,17}$. Posteriormente, dada la recurrencia de hidrotórax, se decidió el manejo con TIPS, logrando una mejoría de la calidad de vida del paciente.

El hidrotórax hepático se desarrolla en 5\%$12 \%{ }^{18}$ de los pacientes con cirrosis e hipertensión portal y su recurrencia es frecuente, a pesar del tratamiento médico. Esta condición está asociada a la presencia de defectos en el diafragma, favoreciendo al paso de líquido desde la cavidad peritoneal ${ }^{2}$. El manejo médico genera un gran impacto clínico, ya que los pacientes son sometidos a toracocentesis a repetición exponiéndose a los riesgos de dicha intervención. En este contexto ha surgido el uso de TIPS.

En el caso descrito, el éxito con el uso de TIPS nos confirma el origen del hidrotórax en el contexto de una cirrosis y sería explicado por la disminución de la presión portal producida por este. En un estudio reciente ${ }^{18}$ se describe una respuesta de $73 \%$ con el uso de TIPS en pacientes con cirrosis e hidrotórax, siendo la complicación más frecuentemente asociada la encefalopatía hepática en 66\%. Otras complicaciones descritas son el sangrado, falla hepática y oclusión. En rela- 
ción a esta última, existen tasas descritas de $80 \%$ dentro del primer año postinserción con stents no recubiertos y con stents recubiertos con PTFE las tasas de permeabilidad descritas van de $74 \%$ a $41 \%$ a 2 años postinserción ${ }^{19}$.

En suma, ante la presencia de un fluido con aspecto lechoso en un paciente con antecedentes de cirrosis, debemos considerar como diagnóstico QA/QT, descartando otras etiologías. Iniciar un manejo precoz, individualizado y escalonado, según las características del paciente. Otorgar un buen soporte nutricional con las medidas previamente mencionadas y lograr reducir la hipertensión portal subyacente.

\section{Referencias}

1. Al-Busafi S, Ghali P, Deschenes M, Wong P. Review Article, Chylous Ascites: Evaluation and Management. ISRN Hepatology 2014.

2. Romero S, Martín C, Hernández L, Verdu J, Trigo C, Pérez-Mateo M, et al. Chylothorax in cirrhosis of the liver: analysis of its frequency and clinical characteristics. Chest 1998; 114: 154-9.

3. Cárdenas A, Chopra S. Clinical reviews: Chylous Ascites. Am J Gastroenterol 2002; 97 (8): 1896-900.

4. Siram K, Meguid RA, Meguid MM. Nutrional support in adults with chyle leaks. Nutrition 2015; 281-6.

5. Smoke A, De Legge M. Chyle Leaks: consensus on management? Nutr Clin Pract 2008; 23 (5): 529-32.

6. Bhardwaj H, Bhardwaj B, Awab A. Transudative chylothorax in a patient with liver cirrhosis: A rare association. Heart Lung 2015; 44 (4): 363-5.

7. Lizaola B, Bonder A, Trivedi HD. Review article: the diagnostic and current management of chylous ascites. Aliment Pharmacol Ther 2017; 46: 816-24.

8. Itkin M, Nadolski GJ. Modern Techniques of Lymphan- giography and Interventions: Current Status and Future Development. Cardiovasc Intervent Radiol 2018; 41 (3): 366-76.

9. Sirvent M, López P, Martínez M. Soporte nutricional y tratamiento con octreótide del quilotórax. Nutr Hosp 2010; 25: 113-9.

10. Dugue L, Sauvanet A, Farges O, Goharin A, Le Mee J, Belghiti J. Output of chyle as an indicator of treatment for chylothorax complicating esophagectomy. Br J Surg 1998; 85: 1147-9.

11. Machado JD, Suen VM, Marchini JS. Is oral nutrition therapy effective for the treatment of chylothorax? A case report. Nutrition 2008; 24: 607-9.

12. Büttiker V, Fanconi S, Burger R. Chylothorax in children. Guidelines for diagnosis and management. Chest 1999; 116: 682-7.

13. Aalami OO, Allen DB, Organ CH Jr. Chylous ascites: A collec- tive review. Surgery 2000; 128: 761-78.

14. Ramos W, Faintuch J. Nutritional management of thoracic duct fistulas: a comparative study of parenteral versus enteral nutrition. JPEN 1986; 10: 519-21.

15. Al-Zubairy S, Al-Jazairi A. Octreotide as a Therapeutic Option for Management of Chylothorax. Ann Pharmacother 2003; 37: 679-82

16. DePew ZS, Iqbal S, Mullon JJ, et al. The role for tunneled indwelling pleural catheters in patients with persistent benign chylothorax. Am J Med Sci 2013; 346 (5): 349-52.

17. Jiménez $\mathrm{CA}$, Mhatre $\mathrm{AD}$, Martínez $\mathrm{CH}$, et al. Use of an indwelling pleural catheter for the management of recurrent chylothorax in patients with cancer. Chest 2007; 132 (5): 1584-90.

18. Campos S, Gomes D, Sofia C. Transjugular intrahepatic portosystemic shunt in refractory hydrothorax - a contribution to an unexplored indication. Eur J Gastroenterol Hepatol. 2016; 28: 661-6.

19. Bhardwaj R, Vaziri H, Gautam A, et al. Chylous Ascites: A Review of Pathogenesis, Diagnosis and Treatment. J Clin Transl Hepatol 2018; 6 (1): 105-13. 